\title{
Influencia de las expectativas de familias y profesorado en el logro educativo y social de los estudiantes
}

\author{
Carmen Gil-del-Pino ${ }^{1}$ \\ ORCID: 0000-0002-8062-2250 \\ Sonia García-Segura ${ }^{1}$ \\ ORCID: 0000-0003-2928-3334
}

\section{Resumen}

Desde los años cincuenta del siglo XX existe un debate sobre si el concepto que los profesores construyen de sus alumnos está relacionado con la extracción social de estos y con el grado de éxito educativo alcanzado. Si así fuera, y son numerosas las investigaciones que apuntan en este sentido, la genialidad de los de capa baja, al no ser percibida ni concebida por sus docentes, quedaría sepultada antes de despuntar. Y si, además, los padres de dichos estudiantes tampoco creen en sus potencialidades ni vislumbran horizontes para ellos, tenemos ya configurada la injusta situación que soportan. Este trabajo persigue indagar sobre el fenómeno en cuestión partiendo de un diseño de corte cualitativo. Este tipo de metodología admite que las creencias, las mentalidades, los mitos, los prejuicios y los sentimientos, entre otros, son elementos de análisis y, por tanto, producen conocimiento sobre la realidad humana. Para ello, se han realizado entrevistas personales a veinticuatro sujetos (doce docentes y doce padres de alumnos), tomados de cuatro centros de Educación Primaria y Secundaria de Córdoba (España). Se trata, en definitiva, de realizar una aproximación empírica al papel de la escuela y la familia en el mantenimiento de la desigualdad de oportunidades a través del análisis del discurso. Los análisis descriptivos señalan la relación entre expectativas, clase social y rendimiento académico, relación que puede atemperarse o diluirse con la experiencia, la reflexión sobre la práctica y la formación permanente.

\section{Palabras clave}

Expectativas - Logro educativo - Extracción social - Desigualdad social - Formación del profesorado.

1- Universidad de Córdoba. Córdoba, España. Contactos: ed1gipim@uco.es; sgsegura@uco.es. 


\section{Influence of the expectations of families and teachers on educational and social achievement of students}

\section{Abstract}

Since the fifties of the twentieth century, there has been a debate about whether the concept that teachers construct of their students is related to the social extraction of the latter as well as their degree of educational success. In that case, and there are numerous researchers that point in this direction, the genius of those students from lower social class backgrounds, which is not perceived or conceived by their teachers, would be buried before it can stand out. Moreover, even if the injustice that falls on them as class inheritance is already intact, the situation may be aggravated by the fact that the parents of these students do not believe in their potential or envision brilliant horizons for them. This paper pretends about the phenomenon in question, designing it from a qualitative research. This type of methodology admits that beliefs, mentality, myths, prejudice and the emotions among others, are elements of analysis to be able to get the personal reality. For that purpose, personal interviews have been conducted with twenty-four subjects (twelve teachers and twelve parents of students) from four primary and secondary schools in Córdoba (Spain). This paper is presented as an empirical approach to the role of school and family in the maintenance of inequality of opportunities through discourse analysis. Descriptive analyses indicate the existence of a relationship between expectations, social class and academic performance which can be tempered or diluted with experience, reflection on practice and ongoing training.

\section{Keywords}

Expectations - Educational achievement - Social extraction - Social inequalities - Teacher training.

\section{Introducción}

En el bloqueo del connatural perfeccionamiento de la persona señalamos con dedo acusador a dos instituciones. La primera es la escuela, que, tal y como afirman los sociólogos Bourdieu y Passeron, tiene dos funciones, una "[...] técnica de producción y de certificación [...] (del saber) y una [...] social” (1981, p. 224) de clasificación y separación de los sujetos. Así, a través de las calificaciones académicas, la institución escolar selecciona a unos alumnos y rechaza y elimina a otros. Pero aún hay más, porque los seleccionados resultan ser los de alta extracción social y los rechazados los de baja. Y no por casualidad. Son muchos los estudios que lo confirman.

Ya en los años cincuenta, Becker (1952) encontró que el profesorado construía su imagen de alumno ideal a partir del prototipo de clase media-alta. Dieciocho años más 
tarde, Rist (2000) vuelve a poner de manifiesto que este alumno ideal reúne una serie de características vinculadas a criterios de clase social que son necesarias para el éxito educativo. En el mismo sentido se pronuncia Van Houtte (2011) cuando afirma que el profesorado tiende a considerar que los estudiantes de elevado estatus socioeconómico obtienen mejores rendimientos, son más inteligentes y ponen más esfuerzo en las tareas escolares que los de bajo.

De acuerdo con los presupuestos anteriores cabe decir que la institución escolar orienta a sus alumnos hacia trayectorias académicas, laborales y sociales diferentes en función de sus orígenes, disponiendo y canalizando el éxito solo de los que proceden de la cultura dominante, por lo que habremos de estar de acuerdo con Kerbo en que "[...] el encauzamiento sirve para reforzar las diferencias de clase” (2003, p. 202). Mientras que a los hijos de las élites se les proporciona ilusión por alcanzar los puestos laborales destacados, a los de las clases bajas se les arranca de raíz la esperanza. En este sentido, Subirats asevera que "[...] todo está dispuesto para el triunfo escolar de los que por nacimiento poseen la 'gran cultura'” (1999, p. 175).

El planteamiento expuesto se halla en la línea del postulado por los sociólogos defensores de la Teoría de la Reproducción (BOURDIEU; PASSERON, 1981; BAUDELOT; ESTABLET, 1976; BOWLES; GINTIS, 1976), para quienes la escuela es un aparato ideológico del Estado cuya función es perpetuar la brecha entre las capas de la sociedad y conducirlas hacia los distintos destinos reservados para ellas. Mas ¿qué mecanismos emplea la institución escolar para que la clase social determine el logro educativo de los estudiantes? Son, fundamentalmente, tres: el lenguaje, la cultura, y las expectativas de los profesores. Los dos primeros han sido estudiados, entre otros, por Bernstein (1989) y Mayoral (2005), quienes hallaron que los códigos comunicativos y los contenidos culturales de la escuela están más próximos a los alumnos de clase alta y media que a los de baja. El tercero, por su parte, desempeña un papel trascendental en el resultado académico.

En efecto, varios estudios han mostrado que los docentes tienen pocas expectativas en relación con los niños de bajo estatus social y que el trato que les dan en función de ellas los aboca sin remedio a un bajo rendimiento. Así, la investigación de Dunne y Gazeley (2008) pone de relieve que dificultades de aprendizaje y posición de clase se superponen en el pensamiento de los docentes. Por su parte, Auwarker y Aruguete (2008) confirman las fuertes expectativas negativas del profesorado en relación con sus alumnos de clase social baja y la dejación de responsabilidad sobre su aprendizaje, al considerar que este se halla fuera de su dominio. Como consecuencia de lo anterior, los estudiantes disminuyen su celo y aprenden a rendir poco, lo que los lleva derecho al fracaso y al abandono escolar.

En el mismo sentido se pronuncia Martínez García cuando afirma que "[...] las clases sociales siguen siendo determinantes para dar cuenta del logro educativo de la población” (2007, p. 292). Por su parte, Bernardi y Requena (2010), en su estudio sobre las desigualdades educativas en los alumnos de educación postobligatoria de nuestro país, concluyen que los resultados académicos se segmentan según el origen social y que los de extracción alta tienen más posibilidades de tener una segunda oportunidad en caso de fracaso. 
Claro que hay una segunda institución que influye de manera determinante también en los resultados académicos de los jóvenes. Se trata de la propia familia. Para Coleman (1968), la influencia del contexto familiar en la vida de los niños repercute en el rendimiento académico en mucha mayor medida que la propia escuela, y ello porque, a través del proceso de socialización, les transmite sus costumbres, lenguaje, creencias, valores y aspiraciones educativas. En este sentido, para Rodrigo y Palacios la familia "[...] constituye en sí misma un escenario sociocultural y el filtro a través del cual llegan a los niños muchas de las actividades y herramientas que son típicas de esa cultura" (1998, p. 29) y a través de las cuales adquieren contenidos y procedimientos que llevan en su interior la impronta del contexto en el que han surgido.

Y como quiera que el techo de oportunidades y el desencanto son rasgos característicos de los contextos socioeconómicamente desfavorecidos, estos cristalizan en los jóvenes y pasan a formar parte de su experiencia común, de su visión del mundo, de sus expectativas. Así, aprenden que la escuela es un agente de logro social pero no para ellos. Aprenden asimismo que su éxito en la vida no depende solo del que obtengan en la escuela sino también del capital cultural y social empleado por su familia. Y dado que este es escaso, dedican poco esfuerzo a las tareas académicas, con lo que obviamente obtienen bajos rendimientos. Y es que “[...] el 'interés' que pone un agente (o clase de agentes) en los 'estudios' [...] no depende solamente del éxito escolar real [...] sino también del grado en que su éxito social dependa de su éxito escolar" (BOURDIEU 1993, p. 404). Y los niños de los estratos inferiores saben que el suyo difícilmente va a proporcionarles acceso a posiciones ocupacionales relevantes, que su desventaja social de origen acabará siendo de manera irremediable desventaja laboral.

Además de las bajas aspiraciones que los padres de contextos desfavorecidos inculcan en sus hijos hay que unir su situación de pobreza económica y cultural y sus problemáticas particulares, que pueden suponer la inversión de las conductas que cabe esperar de ellos. La protección, el cuidado, la expresión de afecto, la educación pueden ser sustituidos por el abandono, la negligencia, el maltrato emocional, el abuso sexual, el maltrato físico o cualquier otro tipo de daño infligido a los niños.

Afirmados los planteamientos anteriores convendría reconocer que la clase social en la que se inscribe la familia es un factor determinante del resultado de la escolarización, pues la mayoría de los estudiantes que fracasan procede de las clases inferiores.

Ahora bien, la escuela y la familia son tanto factores de adscripción como de logro de estatus. Pueden reproducir las desigualdades de clase o promover la movilidad social. Por tanto, es absolutamente preciso que padres y profesores crean en la educación y en las potencialidades de sus hijos y alumnos, que funden su particular credo pedagógico dado que, tal y como afirma Ortega y Gasset, el optimismo es un deber: "Preveo un porvenir humano cada vez más henchido de luminosidad" (2004, p. 158), dice textualmente el gran filósofo.

Nuestro propósito en el presente trabajo consiste en hacer aflorar las concepciones implícitas que funcionan tanto en la familia como en la escuela para analizarlas en profundidad y plantear, en su caso, su modificación a la luz de una filosofía humanista y 
humanitaria apoyada en la infinitud del ser humano y, por tanto, en la creencia firme de que todas las personas pueden alcanzar su pleno desarrollo.

\section{Fundamentación teórica}

\section{La estratificación social}

En el estudio de Lenski (1966) sobre la estratificación social se halló que el grado de desigualdad en una sociedad se relaciona con el desarrollo tecnológico y con la cantidad de bienes excedentes, factores generalmente interrelacionados, pues a mayor desarrollo tecnológico mayor cantidad de excedentes. Para Lenski, “[...] en las sociedades más simples o tecnológicamente más primitivas, los bienes y los servicios disponibles se distribuirán en su totalidad o en su mayor parte sobre la base de la necesidad" (1966, p. 46), mientras que "[...] con el avance tecnológico, una proporción creciente de los bienes y servicios disponibles para la sociedad se distribuirán sobre la base del poder” (p. 46).

El texto anterior nos autoriza a afirmar que la estratificación es una característica básica de las sociedades tecnológicamente desarrolladas. En ellas la renta y la riqueza se distribuyen de modo desigual como resultado de procesos políticos que responden a intereses de clase, de lo que se deriva una distribución también desigual de poder, prestigio, productos básicos, educación y salud.

Todas las sociedades complejas se caracterizan, pues, por su orden jerárquico y por la correspondiente desigual distribución de las recompensas materiales y simbólicas. Claro que ello requiere, tal y como afirma Crompton (1993), algún tipo de sistema de significados que lo explique, justifique y mantenga. Así, de la tradicional consideración de la desigualdad como algo designado por la naturaleza o por la divinidad se pasó, con el desarrollo del industrialismo capitalista, a la idea de que era fruto de la diferencia de aptitud y de esfuerzo de las personas, idea que se inscribe en la teoría de Durkheim (1964), para quien la sociedad se asemeja a un organismo biológico en el que cada parte cumple una función y en el que las diferencias repercuten en beneficio del todo, por lo que se debe permitir a los sujetos ocupar las posiciones más acordes con sus talentos y sus méritos.

Claramente, en el origen de tal argumento liberal se halla la noción de una supuesta igualdad de oportunidades. Si todos tienen las mismas, entonces el resultado desigual puede considerarse justo, pues refleja las diferencias naturales de talentos y no de procesos sociales minuciosa e interesadamente diseñados y articulados. Las recompensas desiguales constituyen, desde este posicionamiento, "[...] una estructura de incentivos que garantiza que los individuos con talento se esfuercen e innoven, contribuyendo así a la mejora de los niveles materiales de la sociedad en su conjunto" (CROMPTON 1993, p. 25).

Planteamientos tales no solo explican y justifican el persistente sistema de desigualdad económica y social característico de las sociedades competitivas de mercado, sino que también lo legitiman y lo hacen moralmente bueno y, por tanto, incuestionable. A través de ellos el poder inculca en los sujetos una visión del mundo estática y los convence de la inmutabilidad de la historia. Se trata de un pensamiento, dice Freire, "odiosamente fatalista" (1997, p. 26), un pensamiento que suprime la conciencia, la racionalidad y la 
subjetividad de las personas y provoca la acomodación. A fuerza de oír que las cosas no pueden cambiar las personas se convencen de ello y permanecen pasivas. El resultado de su quietud será, lógicamente, la perpetuación del estado de las cosas.

\section{La familia como contexto de socialización primaria}

La familia es el ámbito primero y primordial de desarrollo de los niños. Puede definirse como un sistema social abierto que se constituye y mantiene en base a unos fuertes lazos afectivos y en el que ocurren transacciones unidireccionales, bidireccionales y jerárquicas. Para Rodrigo y Palacios la institución familiar es

[...] la unión de personas que comparten un proyecto vital de existencia en común que se quiere duradero, en el que se generan fuertes sentimientos de pertenencia a dicho grupo, existe un compromiso personal entre sus miembros y se establecen intensas relaciones de intimidad, reciprocidad y dependencia. (1998, p. 33).

Una función básica que la célula familiar lleva a cabo, junto a la supervivencia física (reproducción y crianza) y la educación (formación integral), es la socialización, una socialización primaria a través de la cual introduce en los vástagos componentes afectivos, normativos y cognitivos, quienes los incorporan como si de un orden natural de las cosas se tratase. Así, mediante tal función se interioriza lo que se ha construido históricamente, se aprenden los papeles a desempeñar en el escenario social y se asumen actitudes y pautas de conducta con un significado compartido. Para ello, la familia emplea un proceder o estilo de crianza que, según Jorge y González (2017), es el resultado de una multiplicidad de factores tales como la cantidad de hijos, el género y la ubicación ordinal de cada uno, la salud y la apariencia física, el contexto social, cultural y religioso, etcétera. Y si en España durante la dictadura franquista dominaba el modelo parental autoritario, con la democracia ganó fuerza el permisivo, propio de unos padres "[...] tolerantes, que llegan a autorizar casi todo; no exigen mucho a sus hijos o hijas y les permiten que sean ellos mismos quienes controlen sus propias acciones" (JORGE; GONZÁLEZ, 2017, p. 47).

Para Chomsky (2003), el tiempo que los padres pasan con sus hijos se ha reducido drásticamente. Pero es que, además, se ha reducido también el de alta calidad, que es aquel durante el cual los padres no hacen nada más que estar con los hijos esforzándose por conocerlos a fondo, aceptándolos plenamente y ayudándoles a conocerse y a aceptarse, valorando más su esfuerzo que los logros alcanzados, mostrándoles su apoyo incondicional, dándoles unos valores y unas normas, educándolos, en suma.

En esta misma dirección se mueven los estudios sobre diferencias en la dedicación a los hijos en función del nivel socioeducativo de los progenitores. Para Cebolla, Radl y Salazar (2016), este, junto al grado de participación familiar en la escuela, repercute en el logro de habilidades por parte de los pequeños. Los referidos autores encuentran que la Educación Infantil produce efectos positivos en los niños en general, pero estos son menores en aquellos que tienen padres más educados o más involucrados, lo que apunta al alto valor de la relación paterno-filial. 
A lo anterior hay que añadir que los padres de contextos desfavorecidos presentan ideas confusas que les hacen realizar prácticas contrapuestas. Son los padres paradójicos, en terminología de Rodrigo y Palacios (1998), que pueden pasar del más exagerado proteccionismo al abandono y permisividad más absolutos. Todo depende de las circunstancias que rodeen los acontecimientos.

Hemos de referirnos también al hecho de que, frente al modelo de familia clásica cuyo núcleo era el matrimonio heterosexual, está surgiendo una amplia variedad de estructuras familiares que conllevan una adaptación de los estilos educativos en el hogar. El reconocimiento legal del matrimonio homosexual, la progresiva incorporación de la mujer al ámbito público y la del hombre a las tareas domésticas, el aumento de la edad para la maternidad y la paternidad, el acceso cada vez más prematuro de los niños al sistema escolar son algunos indicadores relevantes de la transformación actual de la familia, indicadores que colocan a las sociedades ante un futuro incierto, si bien algunos estudios señalan que los nuevos modelos familiares posibilitan la formación de marcos conceptuales más amplios e igualitarios (OLIVA et al., 2014).

Recapitulando lo expuesto hasta aquí, cabe afirmar que los niños actuales reciben la influencia de los modelos presentados por los medios de comunicación de masas ante la considerable dejadez de los progenitores. Personajes jóvenes, bellos, ricos y famosos constituyen los patrones de la conducta infantil. Los valores materialistas han arrinconado a los culturales, que pierden importancia cuando los prototipos que orientan el comportamiento triunfan sin ellos. Alcanzar riqueza, prestigio y estatus han alcanzado en la sociedad de mercado la condición de metas últimas, pero la creciente variabilidad familiar dota a la sociedad de la esperanza de unas futuras generaciones más abiertas y democráticas.

\section{Escuela y estructura social}

La importancia socializadora de la escuela, en contraste con una apreciación de ella como estrictamente transmisora de contenidos, ha sido evidenciada por los sociólogos de la educación, quienes han contribuido a ampliar el análisis de los procesos e interacciones que tienen lugar en la misma. Para Fernández Enguita (1990), la institución escolar no es un simple vehículo para la transmisión y circulación de ideas sino también un escenario de prácticas sociales. Constituye el segundo espacio de socialización del niño. Lleva a cabo una socialización secundaria que prolonga y diversifica la de la familia. Se trata de una socialización que no precisa estar asociada a componentes emotivos y que hace posible la vida colectiva. Para Berger y Luckman es "[...] la inducción comprensiva (global) y coherente de un individuo a participar del mundo objetivo de una sociedad o de alguno de sus sectores" (1988, p. 185).

Del examen de la literatura científica sobre el papel de la escuela en la sociedad se desprende una tensión dialéctica entre reproducción y cambio. Mientras que para unos la institución educativa por excelencia cumple la tarea de mantenimiento del entramado social, para otros es la principal fuerza motriz de la transformación del estado de las cosas.

Por lo que atañe a la función de conservación de la sociedad, Bourdieu y Passeron (1981) hacen una crítica feroz de la institución escolar, pues consideran que la escuela 
es un subsistema social creado para resguardar tanto las estructuras sociales como las culturas dominantes, a las que consagra como legítimas con el consiguiente debilitamiento o eliminación de las subordinadas. Sirve, pues, de manera específica e insustituible, a las estructuras sociales, a las que mantiene inalterables, pues, al seleccionar significantes y significados que proceden de la cultura hegemónica y que no obedecen a ningún principio universal, solo canaliza el éxito de los sujetos pertenecientes a esta. Dicho con mayor claridad: la escuela legitima e impone la lengua y la cultura dominantes y, a la vez, sanciona en términos de déficits el capital lingüístico y cultural de otras capas sociales. Utiliza dos mecanismos para ello: la arbitrariedad cultural, o selección de significados gratuita e interesadamente, y la violencia simbólica, o imposición de dichos significados ocultando las relaciones de fuerza que los gobiernan.

Explicar el alcance de lo que implica este planteamiento requiere percibir, previamente, que los teóricos de la reproducción niegan la viabilidad de cualquier intento de cambio social desde la escuela, que solo puede reproducir las condiciones para la persistencia de lo establecido, como las relaciones de clase, que son el resultado de la acción pedagógica. De este modo el sistema, fortalecido, se prolonga indefinidamente.

$\mathrm{Al}$ repasar la literatura científica de los años setenta y ochenta del siglo XX llama la atención la gran cantidad de sociólogos que abrazaron la corriente reproduccionista, que llevaba a afirmar que ninguna teoría o práctica educativa podía cambiar las desigualdades sociales (FLECHA, 1997). Así, las teorías que defienden la función educativa de la escuela y que conceden protagonismo a la agencia humana en la mejora de la sociedad desbordan actualmente a las que se fijan solo en su función conservadora. El vuelco formativo producido en España desde mediados del siglo pasado (GARRIDO MEDINA; CHULIÁ, 2005) y su repercusión en la movilidad social ascendente constituyen un fundamento empírico firme para los teóricos que ven en la educación el único modo de reducir y eliminar fenómenos como la pobreza, la marginación, la enfermedad, el analfabetismo, la violencia, el fracaso escolar, la delincuencia, la injusticia, etcétera. Lo que es una sociedad, lo que son sus miembros, y el porvenir de una y de otros "[...] no son cosas que puedan pensarse al margen de lo que ha sido y es el sistema educativo en esa sociedad" (GIMENO SACRISTÁN, 1999, p. 32). De él depende una sociedad más justa, pues puede vulnerar las poderosas relaciones entre origen y destino social.

Para ello, consideramos prioritario el cambio de modelo de organización vigente en los establecimientos educacionales, un modelo mecánico que se remonta a mediados del siglo XIX cuando el objetivo era formar a individuos para que se incorporaran al mundo industrial. En pleno siglo XXI se precisa un cambio organizativo que permita preparar al alumno para el mundo que viene, un cambio desde dentro y colectivo que induzca a los implicados a la búsqueda autónoma de sus propios objetivos de desarrollo y mejora, como han puesto de manifiesto las experiencias y la literatura actual sobre "[...] comunidades profesionales de aprendizaje" (ESCUDER0, 2009, p. 7). El liderazgo pedagógico es otro factor crítico en la mejora de la educación, un liderazgo no restringido al equipo directivo sino compartido o distribuido (HARRIS, 2008). 


\section{Marco metodológico}

Como el fenómeno que pretendemos estudiar tiene un carácter cognitivo, consideramos conveniente utilizar una metodología cualitativa. Esta exige el roce y la esencial cercanía entre el investigador y los sujetos investigados para sondear sus percepciones, sus expectativas y valores, su calidad humana, y origina datos descriptivos como son las palabras, los textos escritos, las conductas, etc. El trabajo cualitativo facilita, pues, un acercamiento intensivo a los fenómenos y nos ubica directamente en la perspectiva de los actores, en sus percepciones y valoraciones de la realidad, en sus expectativas, su visión del mundo y su conciencia.

Así, analizaremos el discurso de los sujetos de investigación para, a través de él, llegar a los distintos significados y representaciones mentales que tienen de la realidad, una realidad compleja constituida por aspectos ocultos como son sus creencias, sus sentimientos, sus intenciones, sus deseos, etcétera. Con tal modo de proceder los hallazgos solo podrán constituir una aproximación a la realidad, que siempre será infinitamente más rica, más amplia y más complicada.

\section{Objetivos de la investigación}

Este trabajo es un intento de descubrir en qué medida factores cognitivos como las ideas implícitas de padres y profesores sobre las posibilidades de sus hijos y alumnos se erigen cual auténticas realidades e impiden que estos desarrollen todas sus potencialidades. Nuestra principal pretensión consiste en explorar el pensamiento de padres y profesores para descubrir las esperanzas que depositan en los niños y calibrar la posible influencia de estas en su éxito o fracaso académico.

\section{Diseño}

El presente trabajo se centra en la perspectiva cualitativa y en el uso de la entrevista como la técnica que mejor puede servir a nuestras intenciones y responder a los interrogantes planteados. Sin duda, este procedimiento nos ayudará mejor que ningún otro a conocer la realidad investigada, pues tiene la virtualidad de extraer las representaciones e impresiones subjetivas de los sujetos de investigación.

\section{Contexto y participantes}

La muestra del presente estudio está integrada por veinticuatro sujetos distribuidos de la siguiente forma:

- Tres docentes y tres padres del Centro de Educación Infantil y Primaria Al Andalus, ubicado en el barrio de clase media Poniente de la ciudad de Córdoba.

- Tres docentes y tres padres del Instituto de Educación Secundaria Maimónides, ubicado en pleno centro de la ciudad de Córdoba. 
- Tres docentes y tres padres del Instituto de Educación Secundaria La Soledad, situado en Villafranca de Córdoba, pueblo que está a 27 kilómetros de la capital cordobesa.

- Tres docentes y tres padres del Instituto de Educación Secundaria Santa Rosa de Lima, ubicado en el barrio de clase baja Campo de la Verdad de la ciudad de Córdoba.

Los criterios que se tienen en cuenta para seleccionar a los participantes son la etapa educativa -Infantil, Primaria y Secundaria- y el contexto -urbano (centro, barrio de clase media y barrio de clase baja) y rural -, con objeto de contar con profesores y padres desde Infantil hasta Secundaria Postobligatoria y de zona rural, del centro urbano y de barrios de distinta consideración social.

En referencia a las condiciones profesionales de los docentes, los de los cuatro centros soportan, y en mayor medida los de los ubicados en barrios de clase media y en el centro de la capital, estructuras organizativas poco flexibles y jerárquicas, escasez de recursos, elevada ratio en las aulas, gran diversidad de alumnado y una cultura de trabajo poco colaborativa.

\section{Instrumentos y técnicas de recogida de datos}

Nuestro principal instrumento de acopio de información es la entrevista semiestructurada y abierta. Elegimos esta técnica porque permite acceder mejor que ninguna otra a los verdaderos pensamientos y emociones de los sujetos de estudio y establecer relaciones entre ellos. Lógicamente, se adapta y especializa según el tipo de destinatario (padre o docente). Consta de cuatro bloques, cada uno referido a un ámbito, si bien no existe una división radical entre ellos, pues nos interesa volver sobre lo tratado para asegurar la fiabilidad de las respuestas dadas por los sujetos a preguntas importantes, que formulamos de manera recurrente y sin imponer limitación alguna. Son los siguientes:

\section{- Entrevista para profesores (D0C)}
a) Variables sociodemográficas y profesionales
b) Perfil sociocultural del alumnado y expectativas sobre el mismo
c) Vivencias y prácticas en el seno escolar
d) Rendimiento académico

\section{- Entrevista para padres (PROG)}
a) Variables sociodemográficas
b) Expectativas sobre los hijos
c) Vivencias y prácticas en el seno familiar
d) Rendimiento académico 


\section{Procedimiento}

Antes de acceder al campo tuvimos un encuentro con un docente de cada una de las instituciones educativas seleccionadas para nuestro estudio, a quien le explicamos los objetivos del mismo y la necesidad que teníamos de contar con la colaboración de tres docentes y tres padres de su centro. Este docente se ocupó de informar sobre el tema a sus colegas y padres de alumnos y de concertar con los que accedieron a participar en la investigación los detalles de la entrevista como fecha, hora, lugar y modo de realización. Una semana después nos hallábamos en el campo realizándola. Hicimos cuatro visitas, una a cada centro, en cuatro días consecutivos.

El examen de los textos orales producidos por los sujetos constó de dos fases. La primera consistió en transcribirlos, ordenarlos y codificarlos teniendo en cuenta el informante (docente o padre), el tipo de centro (Instituto de Educación Secundaria -IES, o Centro de Educación Infantil y Primaria -CEIP) y el contexto del mismo (urbano -centro, barrio de clase media y barrio de clase baja- y rural). El segundo paso estribó en reunir las respuestas dadas por los sujetos a cada una de las preguntas para, de este modo, leer de forma consecutiva las de todos y proceder a la búsqueda de vínculos entre ellas y a su interpretación. Finalmente digamos que, dado el escaso número de sujetos participantes en nuestra investigación (veinticuatro), no usamos ningún programa informático de análisis cualitativo, sino que procedimos manualmente.

\section{Resultados}

Abrimos nuestra exposición con la respuesta dada por un profesor a la pregunta sobre cómo imagina el futuro de sus alumnos, respuesta que resulta sorprendente pero clarificadora para nuestros propósitos al mismo tiempo: "La gran mayoría (excepto dos o tres de dieciséis) reproducirá los mismos esquemas que sus padres: inestabilidad laboral, paro, baja formación..." (DOC 2 IES RURAL). Estas fueron sus palabras textuales referidas a alumnos de un contexto social deprimido y un rendimiento bajo como él mismo expresa en el siguiente párrafo: "La posición socioeconómica es, por lo general, baja, y no hay ningún hábito de trabajo y esfuerzo; el nivel académico es también muy bajo, con gran desfase; de hecho, toda el aula tiene una adaptación metodológica (DOC 2 IES RURAL).

Por lo que atañe a la pregunta sobre qué alumnos tienen peores calificaciones, este docente responde que, “[...] sobre todo, aquellos niños que tienen un nivel socioeconómico bajo y pertenecen a familias que están rotas o no se han preocupado por ellos hasta el momento”.

En los textos anteriores tenemos ya servidos y en relación tres elementos que aparecian en nuestros interrogantes y en nuestras hipótesis como fundamentales: expectativas sobre alumnos (bajas), clase social de estos (baja) y rendimiento académico (bajo). Si las expectativas implican al ser entero, si no son otra cosa que pensamientos y sentimientos conectados a la acción, lo que este profesor piensa y siente sobre sus alumnos de baja extracción social puede estar cristalizando en ciertos comportamientos como menor atención, tareas menos desafiantes, etcétera. Así, el denso entramado cognitivo elaborado sin fundamento científico alguno con el discurrir de los años se puede estar engastando 
en el currículum oculto, que es el conjunto de actitudes, gestos, exclamaciones, miradas, que opera subrepticiamente sobre los alumnos.

A nuestro juicio, el profesor constituye el elemento de mayor peso en el aprendizaje. $\mathrm{Su}$ actitud, su personalidad, su sensibilidad, su estilo docente, su capacidad de establecer relaciones, su metodología, etc., son, sin duda, factores de rendimiento escolar, pues los estudiantes se activan, o no, a raíz de una determinada actuación docente.

Preguntada otra docente del mismo centro sobre el futuro que adivina para sus alumnos, que ahora son de Formación Profesional Básica, nos dice lo que exponemos a continuación:

Sinceramente, aunque soy una profesora que intenta motivar a sus alumnos mediante todos los medios y estrategia a mi alcance, encuentro a este grupo bastante desmotivado y con una parte de la clase que ha abandonado prácticamente. La Formación Profesional es de madera pero tan solo veo a dos dedicándose a eso. La mayoría está aquí por estar escolarizada y obtener el Graduado. Los veo trabajando en cualquier cosa, pero no siguiendo sus estudios. No soy capaz de vaticinar si alcanzarán éxito en la vida pero lo cierto es que ahora mismo lo veo todo muy oscuro para ellos porque no se esfuerzan ni tienen ilusión. (DOC 3 IES RURAL).

Bajas expectativas, de nuevo, sobre alumnos de extracción social baja que rinden poco, según expresa la propia docente: "Su nivel socioeconómico es bajo y su rendimiento académico negativo. En la segunda evaluación solo tres de los trece aprobaron todo" (DOC 3 IES RURAL).

Claro que la terna en cuestión no es específica de este centro rural, sino que nos la encontramos también en los centros de Córdoba capital tanto del núcleo como de los barrios de los que hemos extraído la muestra. "Mis alumnos son de nivel socioeconómico bajo; no los imagino desarrollando su cien por cien de potencial, ya que ahora tampoco lo desarrollan; su rendimiento es bajo, no hay esfuerzo suficiente por su parte ni hay voluntad de trabajo" (DOC 1 IES UR BAJA). Estas son las contundentes palabras de una profesora de Biología de Secundaria que trabaja en un centro de la periferia de Córdoba y que considera que su formación científica está desperdiciada al igual que la de sus compañeros, si bien reconoce que no posee formación pedagógica: "Tengo algo de formación respecto a la violencia en los centros, aunque, si se refiere a si me considero formada en estrategias de resolución de conflictos, le diría que no; soy Licenciada en Biología y esa es mi especialidad; lo demás se sobrelleva” (DOC 1 IES UR BAJA).

En la frase anterior hemos topado con un aspecto esencial. Asoma claramente la punta del enorme iceberg que, a nuestro juicio, está afectando de manera importante a la educación y que hemos hallado en un número importante de entrevistas. Nos referimos a la formación de los profesores, sobre todo a la de los de Secundaria, más orientada a la adquisición de los conocimientos propios de su materia para transmitirlos fielmente que a su preparación pedagógica o didáctica. Se da, pues, mucha importancia al contenido disciplinar, a la especialización del saber, a la competencia en conocimientos. La capacitación en procedimientos y actitudes, sin embargo, tiene menos importancia. Y es que rige un modelo sucesivo cuatro más uno en el que primero se da la formación científica (cuatro años) y posteriormente la psicopedagógica y práctica (un año). Para Esteve, este modelo “[...] 
presenta el problema de crear una identidad profesional falsa en los futuros profesores de Secundaria, que llegan a imaginarse a sí mismos, profesionalmente, como historiadores o como químicos, cuando en realidad van a trabajar como profesores" (2003, p. 212).

Documentemos ahora posicionamientos más optimistas que los presentados hasta aquí. Examinemos las respuestas dadas por dos docentes a la pregunta en cuestión, que recordemos que buscaba descubrir si consideraban que los alumnos obtendrían éxito en un futuro. Esto es lo que respondió literalmente el primero de ellos: "Veo a mi alumnado con capacidades suficientes para desarrollarse profesionalmente en diferentes campos" (DOC 1 IES UR CENTRO). Y este es el segundo enunciado que queremos mostrar: "Creo que sí. Si no pensara que tienen muchas posibilidades no intentaría enseñar. Creo que todos mis alumnos alcanzarán lo que se propongan y, por tanto, tendrán éxito: cada uno de ellos tiene una capacidad o varias y las podrá desarrollar" (DOC 1 IES RURAL). Diez y veinte años en las aulas respectivamente, reflexión sobre la propia práctica y formación continua: estos son los elementos que integran admirablemente estos profesores, según nos revelaron en la conversación mantenida, y sobre los que nos atrevemos a afirmar, con todas las precauciones que se deseen, que constituyen la base de las altas expectativas que depositan en sus alumnos. Esto fue lo que nos dijeron uno y otra:

Llevo diez cursos académicos en ejercicio y ocho en mi actual centro. Tanto la formación del profesor como el vínculo que se establezca entre este y el alumnado son importantísimos. Es muy importante razonar (DOC 1 IES UR CENTRO).

Llevo veinte años en la profesión y tres en este centro. Reflexiono y me pregunto cuál es mi función y qué debo realmente enseñar a mis alumnos. Intento hablar con ellos porque no conozco otra manera. Realizo algunos cursos y leo. Este año he hecho un curso de Educación Emocional en el centro de Profesores y me ha resultado interesante. Me gustó mucho la perspectiva que nos propusieron, que era exponer a los alumnos nuestras necesidades y pedir que nos explicaran las suyas. También me gustó que nos propusieran partir del amor. Creo que las emociones positivas nos ayudan a aprender más u mejor. Me gustaría seguir esta línea y continuar aprendiendo. (DOC 1 IES RURAL).

Puesto que estos enunciados se comentan por sí mismos, dado que apuntan, de nuevo, al papel de la formación del profesorado en su capacidad de creer en los alumnos y en la secreta potencialidad que encierran, nos limitaremos únicamente a plantear dos interrogantes de naturaleza obviamente retórica: ¿la calidad de la educación depende de la calidad de los profesores?; ¿calidad y formación son una misma cosa?

Pero regresemos a la línea más descriptiva de nuestras reflexiones y examinemos ahora las entrevistas realizadas a los padres. ¿Cómo ven estos el futuro de sus hijos? Una madre de zona rural y profesión ama de casa responde lo siguiente: "El éxito en la vida es muy relativo. Yo me conformo con que haya trabajo y más oportunidades y que sea muy feliz en su trabajo y en su vida" (PROG 2 IES RURAL). Preguntada posteriormente por el rendimiento académico de su hijo contesta que "[...] va bien, aunque podría ir mejor porque es muy vago para ponerse a estudiar, lo deja todo para última hora y no tiene 
hábito de estudio diario". Y en relación a las medidas que toman en casa ante conductas incorrectas es rotunda en la contestación: "Lo castigamos sin móvil".

Si en las entrevistas hechas a profesores encontrábamos unidos tres elementos, a saber, expectativas (bajas), clase social (baja) y rendimiento académico (bajo), en la mantenida con esta madre vuelven a aparecer los tres, pero lo hacen junto a un cuarto que entra en escena. Nos referimos al empleo abundante de las tecnologías de la información y la comunicación, empleo que nos confirman otros padres de alumnos como María del Mar, médica de 52 años que, a la pregunta de a qué dedica su hijo el tiempo de ocio, contesta lo siguiente: "Le gusta mucho la televisión y los juegos en Tablet y pasa muchas horas con ellos". Ante conductas incorrectas declara que le da "[...] un castigo tipo no ver la tele o similar" (PROG 3 CEIP MEDIA).

Es patente, desde luego, que alumnos tanto de Educación Primaria como de Secundaria presentan una forma de vida mediada por los citados artilugios. Tienen dispositivos móviles con conexión a Internet, dispositivos que no son, en principio, ni buenos ni malos. El problema está, según Pérez Gómez (1998), en la diabólica combinación entre medios electrónicos y exigencias del mercado y en el control y la finalidad que tenga el uso de los mismos.

Y es que asistimos a una revolución electrónica sin precedentes donde el espacio y el tiempo, según Gil del Pino, se configuran de una nueva forma, ya que "[...] los intercambios a distancia aumentan a la par que disminuyen las relaciones cara a cara" (2005, p. 107). Las relaciones y la comunicación entre las personas en general y entre los niños en particular se han trasladado, pues, a un nuevo campo, el espacio informático o el espacio en la red, lo que hace que surjan nuevas formas comunicativas y lúdicas que modifican los patrones convencionales.

Otro magnífico ejemplo del fenómeno de concomitancia expectativas-clase socialrendimiento lo hallamos en la entrevista hecha a una madre ama de casa de 47 años de edad. Imagina a su hijo en 2038 "[...] hecho un hombrecito y trabajando en lo que pueda; si no hay un cambio global en nuestro sistema me creo que no alcanzará éxito en la vida" (PROG 3 IES RURAL). Como la progenitora 2 del mismo centro a la que aludíamos más arriba, también esta considera a su hijo “[...] más bien vago..., muy flojo, le cuesta tener obligaciones". Adviértase de inmediato que en su respuesta se halla, junto a unas bajas expectativas sobre el hijo, una atribución claramente externa en cuanto a la responsabilidad sobre el futuro éxito del mismo, que, según ella, no dependerá de variables personales (esfuerzo, interés...) sino sistémicas, lo que puede justificar la pasividad que presenta el chico ante los estudios, pasividad que ella no tiene inconveniente en generalizar: "Lo único que siento es que los niños o adolescentes no tienen motivación por nada y menos por el colegio [...]; veo a esta juventud un poco vaga” (PROG 3 IES RURAL).

Si nos movemos ahora a un contexto urbano de clase media encontramos respuestas como la siguiente: "Es muy difícil predecir si mis hijos tendrán éxito o no en la vida, pero sí creo que una buena educación y una preparación académica adecuada les puede ayudar, aunque también valoro mucho la educación emocional: es muy importante" (PROG 1 CEIP MEDIA). Son las palabras textuales de un padre con estudios universitarios. No solo cree con pleno convencimiento en el papel que juega la formación intelectual en el 
porvenir de su hijo sino también en el de la educación integral. Y no puede ser casualidad, aunque habría que investigarlo a fondo, que el rendimiento de su hijo sea bueno: "En general va muy bien en todas las asignaturas". Hallamos, pues, la terna de elementos ya descubierta antes pero ahora con estos situados en el otro polo: expectativas (altas), clase social (media-alta) y rendimiento académico (alto), lo que documenta el poder del contexto próximo del niño en sus calificaciones escolares y nos permite convenir con el sociólogo Coleman y otros autores (1966) en que el ambiente y el trasfondo familiar, tal y como expresamos en nuestro marco teórico, determinan los resultados obtenidos por los estudiantes en mucha mayor medida que la variable escolar.

\section{Discusión y conclusiones}

Una vez presentados los relatos obtenidos en las indagaciones y depurados con rigor, concluimos, en primer lugar, que gran parte de los docentes entrevistados tiene un concepto de sus alumnos muy relacionado con su extracción social, siendo positivo cuando esta es media o alta y negativo cuando es baja, lo que confirmaría lo que postulábamos en la primera hipótesis y que era que sobre los estudiantes de estatus social bajo recaen bajas expectativas de sus profesores y sobre los de estatus alto expectativas elevadas. Claro que hemos hallado también altas expectativas sobre alumnos de clase baja pero solo en el caso de docentes que se describen como muy reflexivos, con abundante experiencia e instalados en procesos de formación continua, lo que constituye nuestra segunda conclusión. En el estudio se patentiza, pues, la necesidad de fortalecer las estructuras formativas de los profesores para que estos tomen conciencia de lo que está en juego dada la interrelación entre su confianza en los alumnos y el éxito académico de estos, también hallada en nuestra investigación, si bien no hemos podido documentar que ello se produce a través de la conducta verbal y no verbal de los docentes, tal y como postulábamos en la segunda hipótesis, conducta que sería captada por los niños, quienes adaptarían a ella la propia y, por consiguiente, sus resultados académicos, que acabarían siendo como los esperados (tercera hipótesis), lo que tampoco podemos aseverar científicamente con los datos recabados.

Otra conclusión que cabe extraer, la tercera, es que el estrato social de los padres, las esperanzas depositadas en sus hijos y el rendimiento escolar de estos discurren en el mismo sentido, lo que se halla en consonancia con las corrientes científicas analizadas en nuestro marco teórico que postulaban la relación entre extracción social de los estudiantes y probabilidad de éxito en la escuela, y también con la hipótesis primera, aquella en la que planteábamos que sobre los estudiantes de estatus social bajo recaen bajas expectativas de sus profesores y de sus propios padres y sobre los de estatus alto expectativas elevadas. Así, los de clase baja tendrían especiales dificultades para alcanzar buenos resultados, al encarnar las ideas de sus progenitores, pues, como afirman Bourdieu y Passeron (1981), además de los bienes económicos que se transmiten a los hijos se les lega también un capital cultural y una serie de esquemas de percepción, ideas, valores y hábitos.

Como cuarta conclusión señalamos el predominio que entre los niños tiene el universo tecnológico sobre el ecológico, el virtual sobre el real. En efecto, tal y como señalan los 
mismos progenitores, estos sienten preferencia por informarse, comunicarse y divertirse a través de la tecnología, acaso inevitable tras la incorporación al ámbito laboral de las madres y el excesivo tiempo que los hijos pasan solos en el hogar guardados, custodiados y entretenidos por ella. No de otro modo se han creado los entornos virtuales en los que se produce el encuentro con los amigos y colegas para integrarse en los grupos de referencia, como relata Bernete García (2010). Las redes sociales tienen, pues, un gran peso en la vida social de los jóvenes, que conectan a través de ellas con sus iguales y con gente con gustos y actitudes parecidos a los suyos, aunque esto robe tiempo a las relaciones auténticas, a las de verdad, como señala el citado autor. La importancia de tal metamorfosis reside en el hecho de que la tecnología se ha convertido en una herramienta que, ya desde la niñez, genera un nuevo tipo de ser humano, puesto que es la primera escuela del niño, por lo que el pensamiento predominante en sus representaciones mentales será el pensamiento concreto. La idea a la que nos referimos se puede describir, para no alargar excesivamente la discusión, mediante la siguiente síntesis que nos ofrece el autor italiano Sartori: "El lenguaje conceptual (abstracto) es sustituido por el lenguaje perceptivo (concreto), que es infinitamente más pobre no solo en cuanto a palabras sino sobre todo en cuanto a riqueza de significación, es decir, de capacidad connotativa” (1998, p. 48).

Finalmente, nuestra quinta conclusión se refiere al locus de control externo que hemos hallado en un texto oral brindado por una madre de contexto social bajo, que declara que el éxito futuro de su hijo pasa por una transformación social en la que ni ella ni su vástago tienen responsabilidad alguna a juzgar por la frase impersonal que construye y que reproducimos nuevamente para someterla a discusión: “[...] si no hay un cambio global en nuestro sistema me creo que [mi hijo] no alcanzará éxito en la vida". Obsérvense los verbos empleados: "hay" para referirse al cambio general y "alcanzará" para el éxito individual. 0 sea, que el cambio se ha de producir y el éxito será alcanzado por el hijo, pero solo si se produjera tal transformación. Nos hallamos ante lo que Freire (1983) llama pensamiento mágico. Este hace que las personas mantengan unas relaciones espontáneas e ingenuas con los demás y con el mundo. Es un pensamiento fatalista en el que la vida consiste en un discurrir mecánico e inexorable. Es vida, pero no existencia, no vida auténtica; es determinación, pero no posibilidad, ni decisión, ni libertad. Si el vivir está prescrito no puede ser auténtico vivir, porque solo lo es cuando el sujeto decide libre y conscientemente cómo quiere que este sea.

En relación con la prospectiva del estudio llevado a cabo planteamos replicarlo con una muestra mayor extraída de más de cuatro centros y con más sujetos de cada uno de ellos. También sería conveniente ampliar el número de preguntas de la entrevista para así intentar de nuevo extraer aspectos que han quedado ocultos como por ejemplo las conductas verbales y no verbales con las que los padres y profesores podrían estar transmitiendo a los jóvenes las expectativas que tienen sobre ellos, expectativas que nos seguimos arriesgando a decir que acaban haciéndose realidad. Claro que en este punto habría que plantear una triangulación de sujetos más amplia que nos permita confrontar no solo los discursos de los docentes y los de los padres de los alumnos sino también los de estos. De tal modo se introducirian en el estudio los pareceres de todos los implicados de manera que apareciesen, entre otros aspectos, las discrepancias, contradicciones y 
omisiones significativas, lo que permitiría realizar un análisis más completo y exhaustivo del objeto de estudio que despierta nuestro interés.

Otro punto en el que habría que seguir investigando es en el de la formación de los padres y los docentes. Interesaría conocer si esta mejora las expectativas de unos y otros sobre sus hijos y alumnos y si con ella disminuyen las dificultades que encuentran en la atención educativa de estos, con la consiguiente influencia en su interés por el estudio y en su rendimiento académico.

En definitiva, tras este trabajo vislumbramos un horizonte constituido por un conjunto de estudios rigurosos y transferibles a la práctica docente con el objetivo de propiciar un cambio de mirada sobre los estudiantes de clase social baja de modo que estos fuesen vistos con toda la potencialidad que atesoran. Conseguiríamos así que regresaran de su inacción y exprimieran con ímpetu libros y lecciones. Los armaríamos con el más sublime y alto bien, el más preciso y precioso que existe: la educación.

\section{Referencias}

AUWARKER, Amy; ARUGUETE, Mara. Effects of student gender and socioeconomic status on teacher perceptions. Journal of Educational Research, E.E.U.U. , v. 101, n. 4, p. 243-246, 2008.

BAUDELOT, Christian; ESTABLET, Roger. La escuela capitalista en Francia. Madrid: Siglo XXI, 1976.

BECKER, Howard. Social-class variations in the teacher pupil relationship. Journal of Educational Sociology, E.E.U.U, v. 25, n. 8, p. 451-465, 1952.

BERGER, Peter; LUCKMANN, Thomas. La construcció social de la realitat. Barcelona: Herder, 1988.

BERNARDI, Fabrizio; REQUENA, Miguel. Desigualdad y puntos de inflexión educativos: el caso de la educación post-obligatoria en España. Revista de Educación, Madrid, n. extra., p. 93-118, 2010.

BERNETE GARCÍA, Francisco. Usos de las TIC, relaciones sociales y cambios en la socialización de las y los jóvenes. Revista de Estudios de Juventud, Madrid, n. 88, p. 97-114, 2010.

BERNSTEIN, Basil. Clases, código y control. Madrid: Akal, 1989.

BOWLES, Samuel; GINTIS, Herbert. Schooling in Capitalist America. New York: Basic Books, 1976.

BOURDIEU, Pierre. Los poderes y su reproducción. In: VELASCO MAílLO, Honorio; GARCÍA CASTAÑO, Francisco Javier; DÍAZ DE RADA, Ángel. Lecturas de antropología para educadores. Madrid: Trotta, 1993. p. 389-429.

BOURDIEU, Pierre; PASSERON, Jean Claude. La reproducción: elementos para una teoría del sistema de enseñanza. Barcelona: Laia, 1981. 
CEBOLLA, Héctor; RADL, Jonás; SALAZAR, Leire. Preschool education as the great equalizer? A crosscountry study into the sources of inequality in reading competence. Acta Sociológica, México, DC, v. 60, n. 1 , p. 41-60, 2016. Disponible en: <http://journals.sagepub.com/doi/abs/10.1177/0001699316654529>. Acceso en: 25 my. 2018.

CHOMSKY, Noam. La (des)educación. Barcelona: Crítica, 2003.

COLEMAN, James Samuel. The concept of equality of educational opportunity. Harvard Educational Review, Massachusetts, n. 38, p. 7-37, 1968.

COLEMAN, James Samuel et al. Equality of educational opportunity. Washington, D.C.: U.S. Government Printing Office, 1966.

CROMPTON, Rosemary. Clase y estratificación: una introducción a los debates actuales. Madrid: Tecnos, 1993.

DUNNE, Mairead; GAZELEY, Louise. Teachers, social class and underachievement. British Journal of Sociology of Education, London, v. 5, n. 29, p. 451-463, 2008.

DURKHEIM, Émile. The division of labor in society. New York: Free Press, 1964.

ESCUDERO, Juan Manuel. Comunidades docentes de aprendizaje, formación del profesorado y mejora de la educación. Ágora para la Educación Física y el Deporte, Valladolid, v. 10, p. 7-31, 2009.

ESTEVE, José María. La tercera revolución educativa. Barcelona: Paidós, 2003.

FERNÁNDEZ ENGUITA, Mariano. La cara oculta de la escuela. Madrid: Siglo XXI, 1990.

FLECHA, Ramón. Pensamiento y acción crítica en la sociedad de la información. In: GOICOETXEA PIÉROLA, Javier José; GARCÍA PEÑA, Javier. Ensayos de pedagogía crítica. Madrid: Popular, 1997. p. 29-43.

FREIRE, Paulo. A la sombra de este árbol. Barcelona: El Roure, 1997.

FREIRE, Paulo. Pedagogía del oprimido. Madrid: Siglo XXI, 1983.

GARRIDO MEDINA, Luis; CHULIÁ, Elisa. Elementos de contexto para el análisis. In: GARRIDO MEDINA, Luis; CHULIÁ, Elisa. Ocupación, formación y el futuro de la jubilación en España. Madrid: Consejo Económico y Social de España, 2005. p. 43-56. (Estudios; n. 173).

GIL DEL PINO, Carmen. Convivir en la diversidad: una propuesta de integración social desde la escuela. Alcalá de Guadaira (Sevilla): MAD, 2005.

GIMENO SACRISTÁN, José. La educación que tenemos, la educación que queremos. In: IMBERNÓN, Francisco et al. La educación en el siglo XXI: los retos del futuro inmediato. Barcelona: Graó, 1999. p. 29-52.

HARRIS, Alma. Distributed leadership in schools: developing the leaders of tomorrow. London: Routledge y Falmer, 2008. 
JORGE, Elizabeth; GONZÁLEZ, María Cristina. Estilos de crianza parental: una revisión teórica. Informes Psicológicos, Bucaramanga, v. 17, n. 2, p. 39-66, Jul./Dici. 2017.

KERBO, Harold. Estratificación social y desigualdad. 5. ed. Madrid: McGraw Hill, 2003.

LENSKI, Gerhard. Power and privilege. New York: McGraw-Hill, 1966.

MARTÍNEZ GARCÍA, José Saturnino. Clase social, género y desigualdad de oportunidades educativas. Revista de Educación, Madrid, n. 342, p. 287-306, 2007.

MAYORAL, Dolores. La teoría de los códigos: desigualdades lingüísticas en educación. Revista Internacional de Sociología, Madrid, n. 41, p. 109-134, 2005.

OLIVA, Alfredo et al. Family structure and children adjustment in Spain. Journal of Child and Family Studies, E.E.U.U., v. 23, n. 1, p. 10-19, 2014.

ORTEGA Y GASSET, José. Obras completas. Tomo I. Madrid: Taurus, 2004.

PÉREZ GÓMEZ, Ángel. La cultura escolar en la sociedad neoliberal. Madrid: Morata, 1998.

RIST, Rayc. Student social class and teacher expectations: the self-fullfilling prophecy in ghetto education. Harward Educational Review, Massachusetts, v. 70, n. 3, p. 257-301, 2000.

RODRIGO, María José; PALACIOS, Jesús (Coord.). Familia y desarrollo humano. Madrid: Alianza, 1998.

SARTORI, Giovanni. Homo videns: la sociedad teledirigida. Madrid: Taurus, 1998.

SUBIRATS, Marina. La educación del siglo XXI: la urgencia de una educación moral. In: IMBERNÓN, Francisco et al. La educación en el siglo XXI: Ios retos del futuro inmediato. Barcelona: Graó, 1999. p. 171-180.

VAN HOUTTE, Mieke. So where's the teacher in school effects research? The impact of teachers, beliefs, culture and beaviour on equity and excellence in education. In: VAN DEN BRANDEN, Kris; VAN AVERMAET, Gred; VAN HOUTTE, Mieke (Ed.). Equity and excellence in education: towards maximal learning opportunities for all students. New York: Routledge, 2011. p. 75-95.

Recibido en: 25.09 .2018 Revisado en: 18.12.2018 Aprobado en: 13.02.2019

Carmen Gil-del-Pino es doctora en Educación por la Universidad de Córdoba, y miembro del grupo de investigación SEJ-477: Educación, Diversidad y Sociedad (EDISO). Profesora titular en el Departamento de Educación (área Teoría e Historia de la Educación) de la Facultad de Ciencias de la Educación de la Universidad de Córdoba (España). 
Sonia García-Segura es doctora en Antropología Social por la Universidad de Granada y miembro del grupo de investigación SEJ-614: Infancia, Ciudadanía, Democracia y Educación (INCIDE). Profesora investigadora en el Departamento de Educación (área Didáctica y Organización Escolar) de la Facultad de Ciencias de la Educación de la Universidad de Córdoba (España). 